IAU Colloquium 164: Radio Emission from Galactic and Extragalactic Compact Sources

ASP Conference Series, Vol. 144,1998

J. A. Zensus, G. B. Taylor, \& J. M. Wrobel (eds.)

\title{
Sub-mas Jets in Gamma-Active Blazars: Results from High Fre- quency VLBI
}

\author{
T. P. Krichbaum, A. Kraus, K. Otterbein, S. Britzen, A. Witzel \\ Max-Planck-Institut für Radioastronomie, D-53121 Bonn, Germany
}

\author{
J. A. Zensus ${ }^{1}$ \\ National Radio Astronomy Observatory, Charlottesville, VA 22903, USA
}

\begin{abstract}
We report new results from high frequency $(22-86 \mathrm{GHz})$ VLBI monitoring observations of selected blazars. These Gamma-bright sources show pronounced correlated flux density variations over the full electromagnetic spectrum (radio to Gamma-ray bands). From our high-angular resolution images (0.1-0.2 mas), we find increasing evidence for a tight correlation between this activity and the production of new jet components. Here we present results for the 3 sources PKS $0528+134,3 \mathrm{C} 273, \& 0836+710$.
\end{abstract}

0528+134: In 1991 and 1993 two jet components (N1 \& N2) were ejected near times of prominent Gamma-flares (cf. Krichbaum et al. 1995). The $90 \mathrm{GHz}$ light curve (Fig. 1a) shows quasi-periodic variations. The flare of 1993.5 probably is related with a corresponding Gamma-flare. New jet components (N2-N5) seem to appear at times of local minima in the light curve. Close to the core the components move along a bent trajectory (Fig. 1b; for motion further out see Britzen et al., these Proceedings, p. 43). This behavior suggests a geometrical interpretation, eg. within the lighthouse model (Camenzind \& Krockenberger 1992). In contrast to this, the observed steepening of the radio spectrum $\left(\alpha_{22 / 90 \mathrm{GHz}}\right)$ between 1993 and 1996 and the lower amplitudes of the two recent Gamma-flares (peaking approx. $1995.3 \& 1996.5$ ) indicate that the Gamma-activity cannot be explained by geometrical effects alone.

3C 273: Two $86 \mathrm{GHz}$ images (Fig. 2) show pronounced jet expansion between 1994-1995. Combining these images with data obtained at $22 \& 43 \mathrm{GHz}$ results in a tentative component identification, in which the (new) jet components C12, C13, \& C14 were ejected in 1992.5, 1993.4, \& 1994.3, respectively. Within the present uncertainties ( $\Delta t= \pm 0.6 \mathrm{yrs}$ ), it is therefore likely that C13 is related to the prominent Gamma-flare observed in 1993.9 (von Montigny et al. 1997). 0836+710: In Fig. 3a (left) we show the motion of the innermost jet component B3, which was ejected after a correlated Gamma-, X-ray, optical flare peaking early 1992 (Otterbein et al., in prep.). Fig. 3b (right) shows the evolution of the spectral turnover in the total spectrum of $0836+71$ after subtracting the quiescent spectrum. The numbers indicate the time sequence (1992-1993). The observed adiabatic expansion $\left(S_{m} \propto \nu_{m}^{(0.4 \pm 0.2)}\right)$ indicates that the outburst of 1992 has generated an electron spectrum, harder than typical for this source (cf. Qian et al., these Proceedings, p. 93).

\section{References}

Camenzind, M., \& Krockenberger, M. 1992. A6A, 255, 59-62.

Krichbaum, T., et al. 1995. Proc. Natl. Acad. Sci. USA, 92, 11377-11380.

von Montigny, C., et al. 1997. ApJ, 483, 161-177.

${ }^{1}$ Present address: Max-Planck-Institut f. Radioastronomie, Bonn, Germany. 

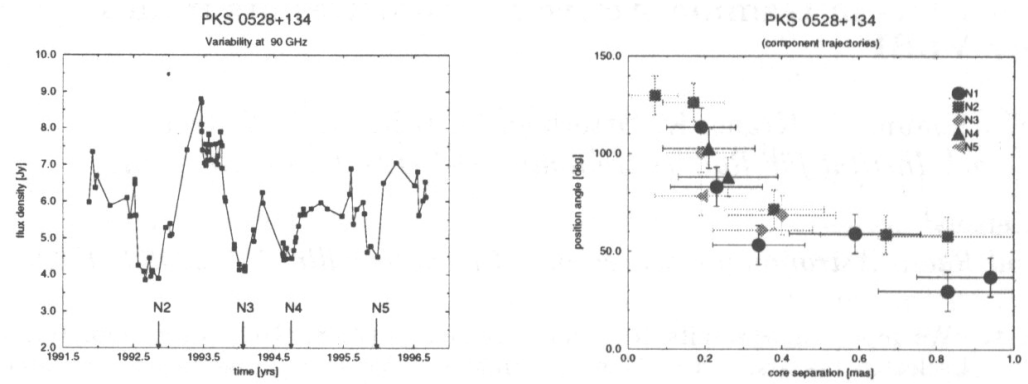

Figure 1. Lightcurve of $0528+134$ at $90 \mathrm{GHz}$ (cf. Ungerechts et al., these proceedings, p. 149). The arrows mark extrapolated ejection times of the new VLBI components. On the right the relative positions $(r, p a)$ of the inner jet components are plotted.
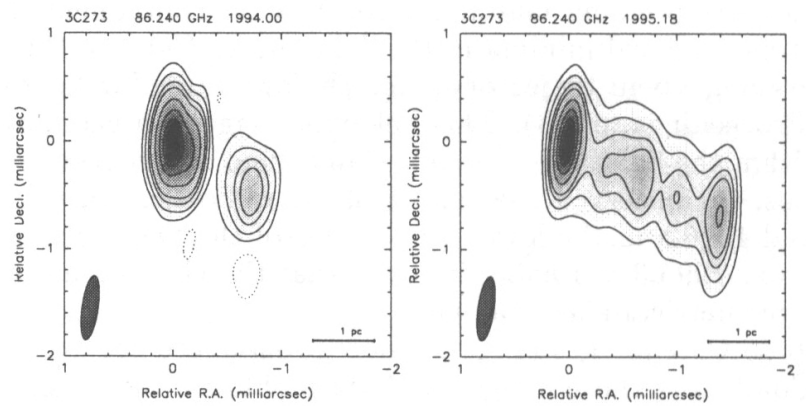

Figure 2. Expansion of the jet of $3 \mathrm{C} 273$ at $86 \mathrm{GHz}$. Both images are restored with a beam of size $0.60 \times 0.15$ mas, $p a=-8^{\circ}$. Contour levels are at $-2,2,5,10,15,30$, $50,70,90 \%$ of the brightness peak of $1.96 \mathrm{Jy} /$ beam (left), respectively $4.84 \mathrm{Jy} /$ beam (right).
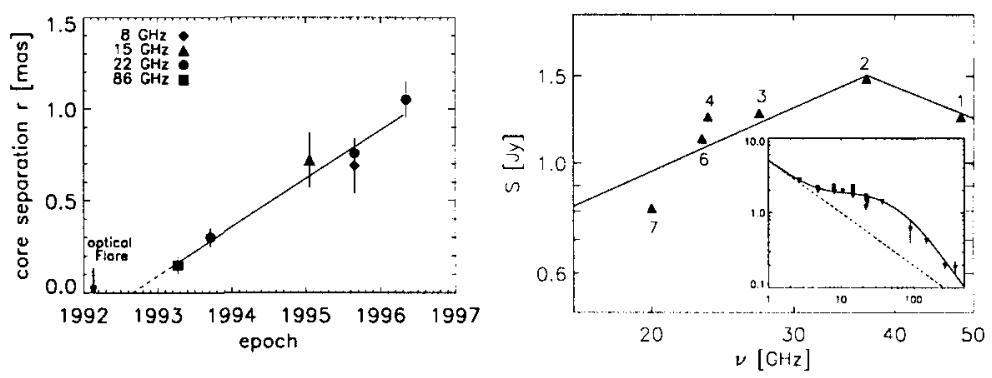

Figure 3. Motion of a new jet component B3, ejected after a correlated Gamma-/Xray/optical outburst observed in the S5 blazar $0836+710$ in 1992.13. The evolution of the spectral turnover is shown on the right, the insert shows a simultaneous spectrum for 1992.35. A data point of 1996.3 is from A. Marscher (priv. comm.) 